\title{
Tunable Multi-Frequency Optoelectronic Oscillator Based on a Microwave Photonic Filter and an Electrical Filter
}

\author{
Jin Zhang
}

Wuhan Electronic Institute

\section{Yalan Wang}

Wuhan Electronic Institute

\section{Xiang Li}

Hubei University

Zhuangzhuang Liu

Wuhan Electronic Institute

Jianghai Wo ( $\nabla$ jianghai_wo@163.com )

Air Force Early Warning Academy https://orcid.org/0000-0001-8500-7989

\section{Research Article}

Keywords: Microwave generation, microwave photonics, optoelectronic devices

Posted Date: May 12th, 2021

DOI: https://doi.org/10.21203/rs.3.rs-488878/v1

License: (9) This work is licensed under a Creative Commons Attribution 4.0 International License. Read Full License

Version of Record: A version of this preprint was published at Optical and Quantum Electronics on July 14th, 2021. See the published version at https://doi.org/10.1007/s11082-021-03061-0. 


\title{
Tunable Multi-Frequency Optoelectronic Oscillator Based
}

\section{on a Microwave Photonic Filter and an Electrical Filter}

\author{
Jin Zhang, ${ }^{1+}$ Yalan Wang, ${ }^{1+}$ Xiang Li, ${ }^{2}$ Zhuangzhuang Liu, ${ }^{3}$ and Jianghai Wo ${ }^{1 *}$ \\ ${ }^{1}$ Department of Microwave Photonics, Air Force Early Warning Academy, Wuhan 430019, China \\ ${ }^{2}$ Hubei Key Laboratory of Ferroelectric and Dielectric Materials and Devices, Faculty of Physics and \\ Electronic Science, Hubei University, 430062, China \\ ${ }^{3} 63795$ troops, PLA, Beijing 100089, China \\ ${ }^{+}$These authors contributed equally to this work \\ *(E-mail)jianghai_wo@163.com
}

\begin{abstract}
We propose and experimentally demonstrate a multi-frequency optoelectronic oscillator (OEO) based on phase-modulation to intensity-modulation (PM-IM) conversion. A microwave photonic filter (MPF) incorporating an optical fiber Bragg grating FabryPerot (FBG-FP) filter and an electrical yttrium iron garnet (YIG) filter in two branches are used to select the oscillation modes of the OEO. By adjusting the wavelength of laser source or the central frequency of YIG filter, two frequencies can be tuned independently within a wide range. The single sideband (SSB) phase noises of the generated frequencies are measured experimentally. Moreover, the OEO shows potential ability to achieve more frequencies oscillation by multiplexing more optical and electrical filters.
\end{abstract}

Keywords: Microwave generation; microwave photonics; optoelectronic devices.

\section{Introduction}


Microwave photonics studies the interaction between microwave and optical signal, whose major functions include photonic generation of microwave signals [1, 2], photonic processing of microwave signals [3-5], optically controlled phased array antennas, and photonic analog-todigital conversion [6]. With the fast development of information industry in recent years, there has been a pressing need for high-frequency signal sources with wideband tunability in a lot of fields such as channelized digital receiver, next generation wireless radio communication [7] and modern radar [8-10]. Optoelectronic oscillator $(\mathrm{OEO})$ is a promising method to generate microwave signals with high-frequency, high-stability, and high spectral-purity [11]. A trend in wireless applications has been developing multi-mode and multi-band operation, particularly for automotive radar applications [12]. However, traditional OEO can only generate one single frequency. Although multi-frequency could be achieved by employing more than one OEO, it will increase cost and complexity of the system, and the coherence of frequencies cannot be guaranteed. Meanwhile, the oscillation frequency is usually fixed, which is determined by weak tunability of the filter.

To solve this problem, many researchers have carried out their works in order to generate more than one frequency simultaneously in terms of the OEO. Jiang et al. demonstrated a dualfrequency OEO by simply adding two electrical filters in parallel branches [13]. However, because of the use of fixed electrical filters, the generated signals were not tunable. Kong et al. employed a polarization modulator (PolM) and a phase-shifted fiber Bragg grating (PS-FBG) to form a dual-frequency OEO [14]. When transverse force was applied, two orthogonally polarized notches of the PS-FBG will be changed because of fiber birefringence, as well as the oscillation frequencies. However, the tunability of the two frequencies are not independent. A tunable multifrequency OEO based on stimulated Brillouin scattering was reported by Pei Zhou [15]. This 
scheme used stimulated Brillouin scattering (SBS) effect to convert phase-modulation into intensity-modulation (PM-IM), and independently tuning of each generated frequency can be achieved by changing the wavelength of the corresponding tunable laser source. Xie et al. used a dual-parallel Mach-Zehnder modulator (DPMZM) to form a dual-frequency OEO with low intermodulation [17]. Independent tunability is achieved by using two parallel electrical filters.

In this letter, we proposed and experimentally demonstrated a multi-frequency OEO scheme based on phase-modulation to intensity-modulation (PM-IM) conversion [18]. The OEO is composed of two filtering branches. One branch employs a microwave photonic filter (MPF) including a tunable laser source (TLS), a phase modulator (PM) a fiber Bragg grating Fabry-Perot (FBG-FP) filter and a photodetector (PD) to achieve PM-IM conversion and select one oscillation frequency. By tuning the wavelength of TLS, oscillation frequency tunability can be achieved. The other branch also incorporates PM-IM conversion, in which a section of single mode fiber (SMF) is used as dispersive device to convert phased modulated optical signal to intensity modulated signal. The oscillation mode is then selected using an electrical yttrium iron garnet (YIG) filter in electric domain, which can be tuned by changing the driving voltage of the YIG filter. Experiments are carried out to demonstrate the feasibility and tunability of our approach. The phase noise performance of two generated signals is also investigated.

\section{Principle of operation}

The configuration diagram of proposed OEO is illustrated in Fig. 1. The OEO consists of a TLS, a PM, an optical coupler (OC), an optical circulator, an FBG-FP filter, an erbium-doped fiber amplifier (EDFA), two PDs, a section of SMF, a variable optical attenuator (VOA), an electric coupler (EC) and a microwave power amplifier (PA). A continuous wave (CW) light from the TLS is sent to the PM. The phase modulated light signal is divided into two branches by a 1:1 
OC. The two branches use optical and electrical filtering methods to filter out two different electrical signals, respectively. Then the signals are combined by an EC and amplified by a PA. Finally, the signals are sent back to the PM to make the oscillation loop closed.

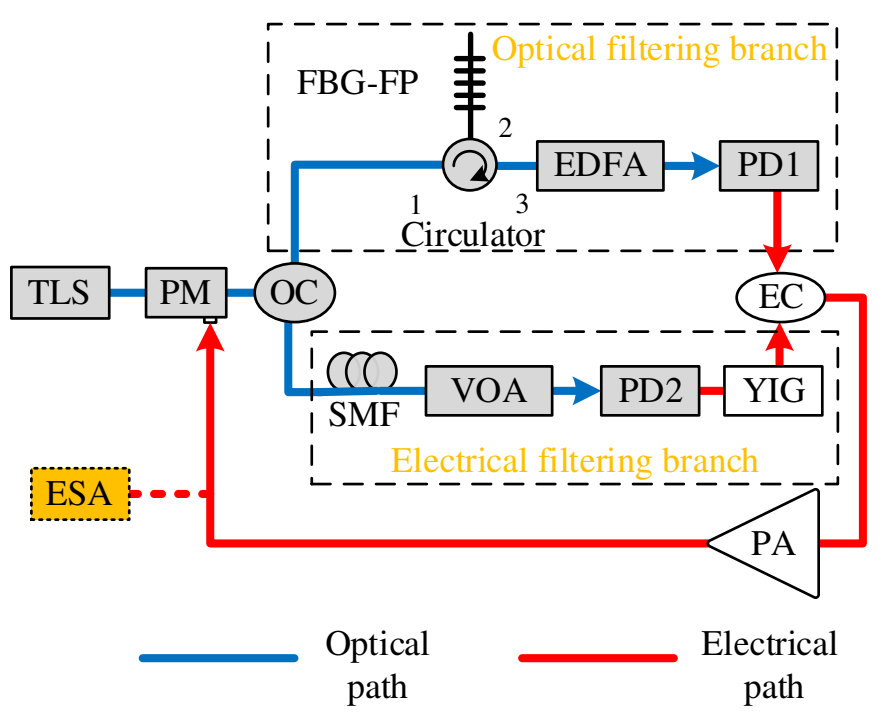

Fig. 1 Schematic diagram of the proposed tunable multi-frequency OEO.

In order to achieve independent tunability, an optical filtering branch and an electrical filtering branch are used to select and tune two different oscillation frequencies. The upper branch in Fig. 1 uses the MPF that consists of the TLS, PM, FBG-FP and PD to determine an oscillation frequency. The operating principle of this MPF is shown in Fig. 2, where the black line refers to the optical carrier. Fig. 2(a) and (b) depict the phase modulated signal before and after the FBGFP, respectively. Assuming the reflection top of FBG-FP is flat, the signal at the output of PD1 is given by [19]

$$
\begin{aligned}
V\left(\omega_{e}\right) & \propto 2 E_{0}^{2} J_{0}(\beta) J_{1}(\beta) \sqrt{r\left(\omega_{0}\right)} \\
& \times\left\{\sqrt{r\left(\omega_{0}+\omega_{e}\right)} \cdot \cos \left(\omega_{e} t+\theta_{1}\right)\right. \\
& \left.-\sqrt{r\left(\omega_{0}-\omega_{e}\right)} \cdot \cos \left(\omega_{e} t+\theta_{2}\right)\right\}
\end{aligned}
$$

where $r(\cdot)$ is the power reflection coefficient of the FBG-FP, $\omega_{e}$ is the angular frequency of the modulating signal, $\omega_{o}$ is the angular frequency of the light wave. $J_{n}(\cdot)$ is the $n$ th-order Bessel 
function of the first kind. $E_{o}$ is the amplitude of the incident light wave. When no first-order sidebands fall into the notch of the FBG-FP filter, we have

$$
\begin{aligned}
\sqrt{r\left(\omega_{0}+\omega_{e}\right)} & =\sqrt{r\left(\omega_{0}-\omega_{e}\right)} \\
\theta_{1} & =\theta_{2}
\end{aligned}
$$

According to Eq. (1), no signal will be detected (like the red sideband in Fig. 2). However, when the notch of FBG-FP filters out one sideband of desired signal (like the blue sideband in Fig. 2), the Eq. (2) and Eq. (3) will be not satisfied. Thus, phase-modulated signal is converted to intensity-modulated signal. A signal is detected by PD, whose frequency is equal to the difference between the notch of FBG-FP filter and the wavelength of optical carrier. In this case, tunability can be easily realized by tuning the wavelength of TLS.

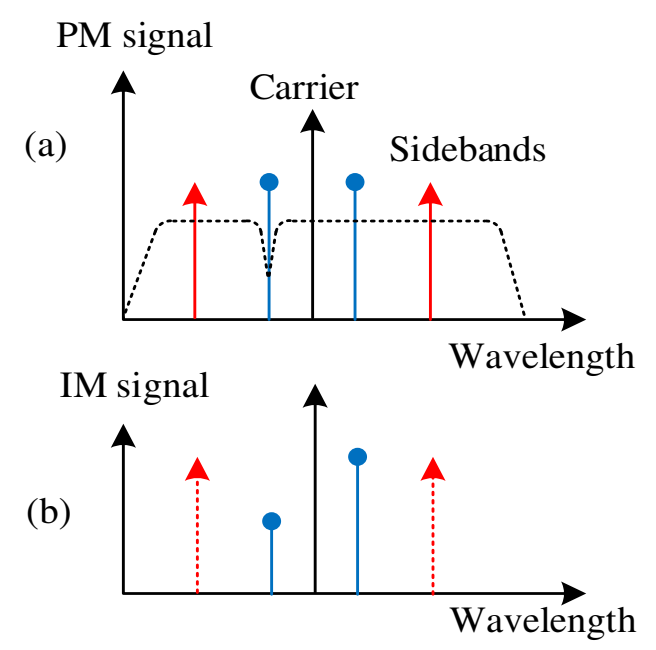

Fig. 2 Operation principle of the optical filtering branch. (a) The phase-modulated signal. (b) The phasemodulated signal after filtered by FBG-FP.

On the other hand, the PM-IM conversion in the lower filtering branch is achieved by using a dispersive device. The operation process of the lower filter branch is shown in Fig. 3. 
It has been demonstrated that the combination of a TLS, a PM, a section of SMF, and a PD form a MPF whose frequency response is shown as the blue line in Fig. 3(a) [20]. The optical field after the SMF can be expressed as

$$
\begin{aligned}
E(t) \propto J_{0} \cos \left(\omega_{0} t+\varphi_{0}\right) \\
+J_{1} \cos \left(\omega_{1} t+\frac{\pi}{2}+\varphi_{1}\right) \\
\quad-J_{1} \cos \left(\omega_{2} t-\frac{\pi}{2}+\varphi_{2}\right)
\end{aligned}
$$

where $\varphi_{0}, \varphi_{1}$, and $\varphi_{2}$ are the phase delays of the spectral components $\omega_{0}, \omega_{1}$, and $\omega_{2}$ induced by the chromatic dispersion of SMF, respectively. The chromatic dispersion of SMF induces different phase delays for different sidebands, changing the phase modulated signal into intensity modulated signal. Finally, the recovered RF signal can be expressed as

$$
E_{R F}(t) \propto \cos \left(\frac{\pi \chi \lambda_{0}^{2} f_{m}^{2}}{c}+\frac{\pi}{2}\right) \cdot \cos \left(\omega_{m} t+\theta\right)
$$

where $\chi$ is the accumulated dispersion of SMF, $\lambda_{0}$ is the wavelength of optical carrier, $f_{m}$ is the frequency of the modulating signal, $c$ is the velocity of light in vacuum, $\theta$ is the phase delay of the recovered signal. It can be seen from Eq. (5), the first notch locates at the dc frequency. The second notch is determined by

$$
\pi \chi \lambda_{0}^{2} f_{m}^{2} / c=\pi
$$

As can be seen, we can obtain a rather wide passband up to tens of gigahertz by choosing a period of SMF with proper length. This passband is wide enough to cover the oscillation turning range. Since the value of optical carrier wavelength is much smaller than the value of modulating signal, the wavelength change of the optical carrier will cause little impact on the passband, according to Eq. (6). In other words, the tuning of the upper filtering branch is independent on the lower one. To realize a narrow band filtering branch, an electrical YIG filter is employed to select 
the oscillation frequency. The YIG filter is a band-pass filter with a bandwidth as narrow as tens of megahertz and a large tunability up to tens of gigahertz.

The frequency response of the YIG filter is shown as the red line in Fig. 3(a). Fig. 3(b) shows the frequency response of the whole lower filtering branch. By adjusting the driving voltage of the YIG filter, the passband can be continuously tuned within a wide range.

(a)

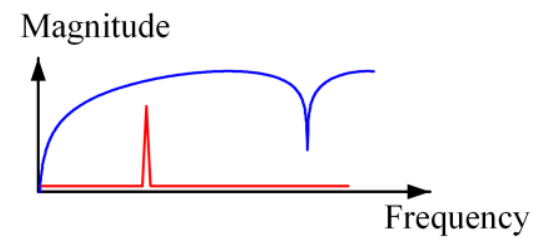

(b)

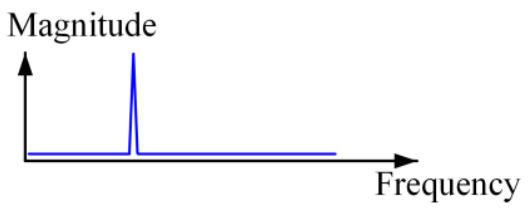

Fig. 3 Operation principle of the electrical filtering branch using a YIG filter. (a) The frequency response of the band-pass MPF (the blue line) and the frequency response of the YIG filter (the red line); (b) The frequency response of the electrical filtering branch.

\section{Experiment results and discussion}

Experiment investigation is carried out according to the setup shown in Fig. 1. A TLS with a maximum output power of $16 \mathrm{dBm}$ is used as the light source. The bandwidth of PM is larger than $30 \mathrm{GHz}$. The FBG-FP fiber filter has a reflection bandwidth of about $0.7 \mathrm{~nm}$ and is composed of two wavelength matched Bragg gratings with appropriate separation. The cavity length of the FBG-FP filter is several millimeters so as to achieve a single notch within the reflection band. The notch wavelength of the FBG-FP locates at $1551.168 \mathrm{~nm}$ with a $3 \mathrm{~dB}$ bandwidth of $90 \mathrm{MHz}$. The SMFs used in the optical and electrical branch are $400 \mathrm{~m}$ and 4000 $\mathrm{m}$, respectively. Two PDs with the bandwidth up to $50 \mathrm{GHz}$ are used for photoelectric 
conversion. The length of SMF is $4 \mathrm{~km}$. A VOA at the input of PD is inserted to make the gain of this filtering branch at the same magnitude with the other one. The central frequency of the YIG filter can be tuned by controlling its driving voltage from $0 \mathrm{~V}$ to $10 \mathrm{~V}$.
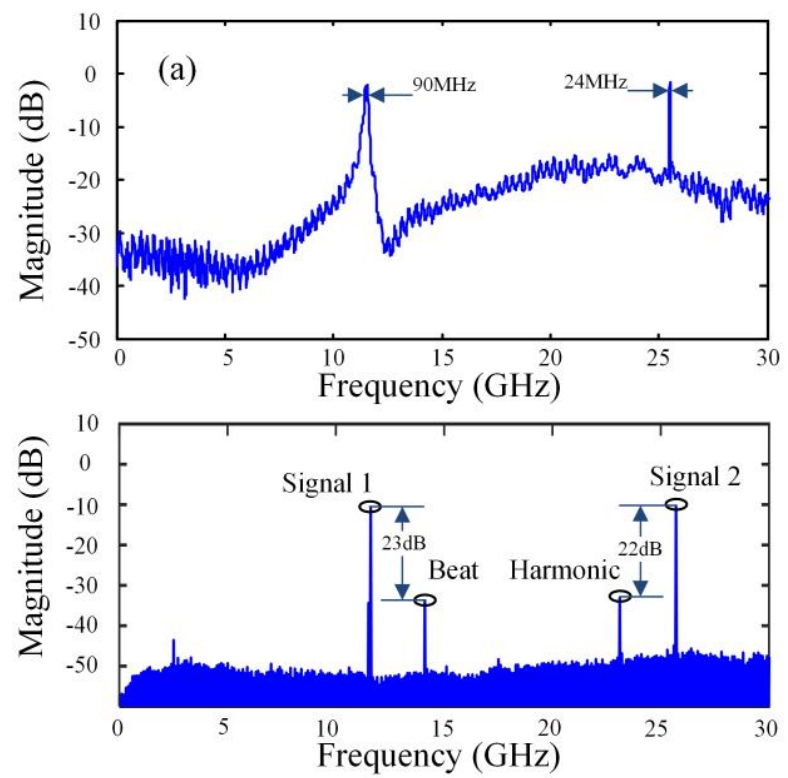

Fig. 4 (a) The frequency response of the dual-frequency MPF; (b) The electrical spectrum of oscillation signals.

Before investigating the oscillation characteristics of the OEO, the open loop frequency response is measured through a vector network analyzer (VNA, Keysight N5244A). Fig. 4(a) shows the measured frequency response with the TLS wavelength of $1551.26 \mathrm{~nm}$ and the YIG driving voltage of $6.2 \mathrm{~V}$. Two frequency peaks locate at $11.58 \mathrm{GHz}$ and $25.52 \mathrm{GHz}$ can be observed. The $3 \mathrm{~dB}$ bandwidths of the two peaks are $90 \mathrm{MHz}$ and $24 \mathrm{MHz}$, respectively.

Then, the OEO loop is closed. Once the EDFA and the VOA are tuned at proper values, two frequencies will oscillate simultaneously, as shown in Fig. 4(b). The generated signals are monitored by an electrical spectrum analyzer (ESA, Keysight N9040B). Mode competition exists in the cavity since both frequencies share the same electrical amplifier. However, when the frequency difference of the two oscillating signals is large enough, two stable oscillating signals 
can be observed by controlling the optical and electrical gain of the OEO loop. Only beat frequency and second-order harmonic signal exist, which can be suppressed to more than $20 \mathrm{~dB}$ lower than the oscillating signals, as shown in the Fig. 4(b). Figure 5 shows the zoomed-in view of the two oscillating signals when the two oscillating frequencies are tuned around $14.99 \mathrm{GHz}$ and 25.66 GHz, respectively. Figure 5(a) depicts the oscillating frequency for the optical branch, in which the mode spacing can be calculated to be $429.31 \mathrm{kHz}$. A sidemode suppression ratio (SMSR) of $43.37 \mathrm{~dB}$ can be observed. Other spurs can also be observed, which are caused by the modes of the electrical branch. Similarly, the mode spacing and the SMSR of the electrical branch are $49.56 \mathrm{kHz}$ and $53.12 \mathrm{~dB}$, respectively. The SMSR of the optical branch is not as high as that of the electrical branch because of the relatively large bandwidth of the MPF. The SMSR performance of the optical branch can be much improved by employing an FBG-FP with narrower notch bandwidth.
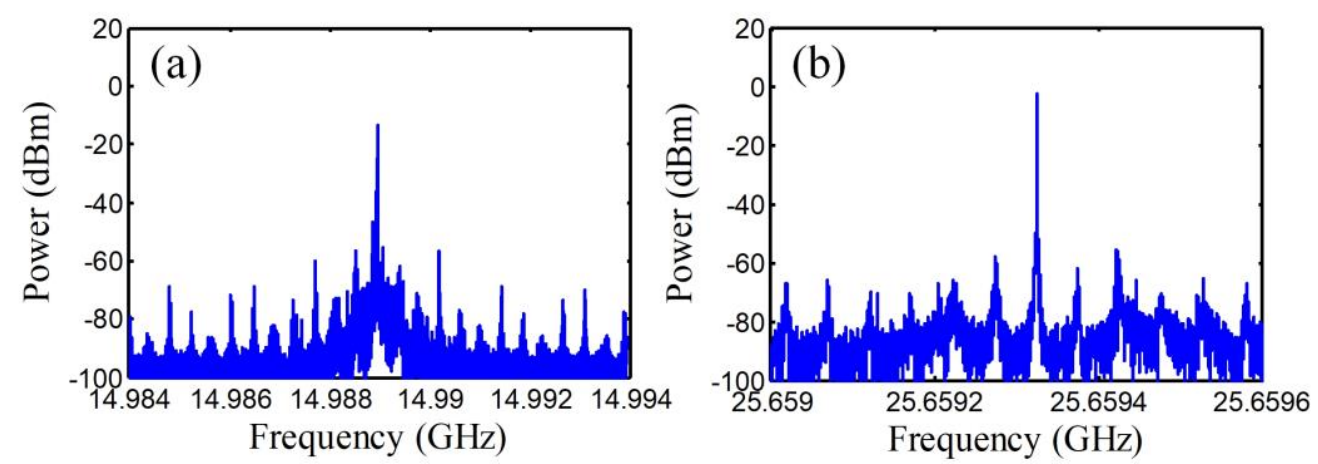

Fig. 5 (a) Zoomed-in view of the oscillating frequency of the optical branch with a span of $10 \mathrm{MHz}$; (b) Zoomedin view of the oscillating frequency of the electrical branch with a span of $600 \mathrm{kHz}$;

To prove the independent tunability of two frequencies, we measured the tunability of one frequency by keeping the other unchanged. First, we fixed the driving voltage of YIG filter at 6.2 $\mathrm{V}$ and increased the wavelength of TLS from $1551.20 \mathrm{~nm}$ to $1551.29 \mathrm{~nm}$. The electrical spectrum in Fig. 6(a) shows that the oscillation frequency of signal 1 increases from $5.81 \mathrm{GHz}$ to 
$18.18 \mathrm{GHz}$, while the frequency of signal 2 keeps stable. The tunning range of signal 1 is limited by the reflection bandwidth and the notch position of FBG-FP filter. To improve the tuning range, FBG-FP filter with broader reflection bandwidth can be used. Fig. 6(b) demonstrates the linear relationship between the frequency of signal 1 and the wavelength of TLS. Since the wavelength tunning resolution of the $\mathrm{LD}$ is $1 \mathrm{pm}$, the frequency tuning resolution of the signal generated by the optical branch should be $125 \mathrm{MHz}$. Furthermore, more PS-FBGs with different central wavelengths can be cascaded to achieve multi-frequency oscillation for the optical branch when a multi-wavelength laser source is used.
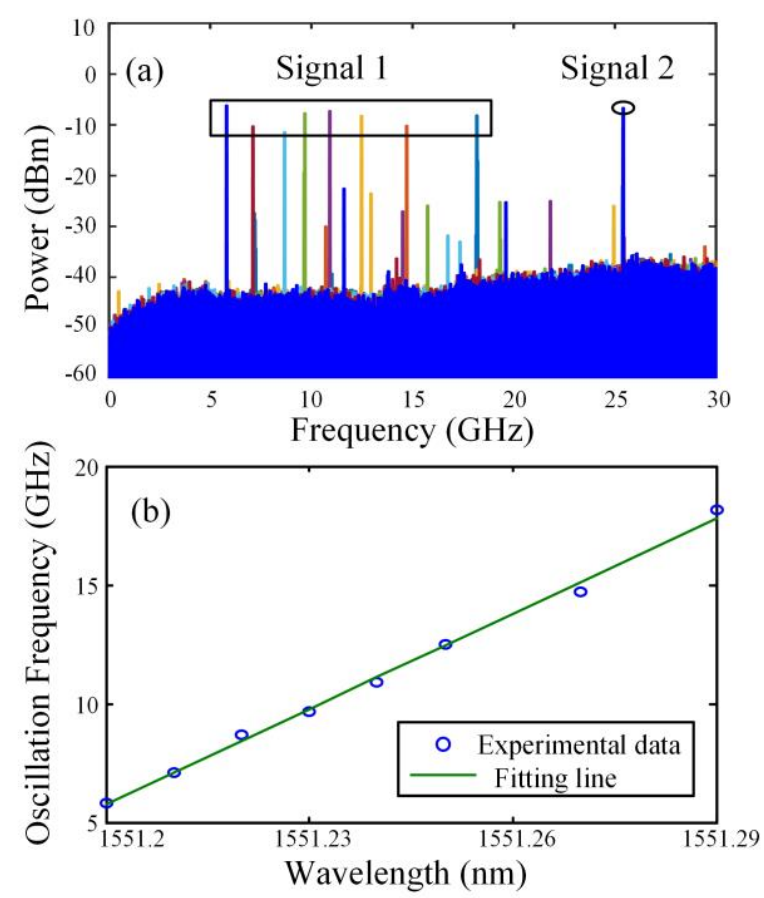

Fig. 6 (a) The superimposed spectra of the generated signals under different TLS wavelengths; (b) The relationship between the frequency of signal 1 and the TLS wavelength.

Then the wavelength of TLS is fixed at $1551.26 \mathrm{~nm}$, and the driving voltage of the YIG filter is increased from $2.9 \mathrm{~V}$ to $5.9 \mathrm{~V}$ in order to demonstrate the electrical tunability. The frequency tuning resolution of the YIG filter is about $50 \mathrm{MHz}$. Fig. 7(a) shows the superimposed spectra of the proposed OEO. As depicted in Fig. 7(a), the signal 1 keep unchanged at $11.91 \mathrm{GHz}$ when 
signal 2 is tuned over a range from $13.11 \mathrm{GHz}$ to $24.31 \mathrm{GHz}$. The relationship between the frequency of signal 2 and the driving voltage of the YIG filter is shown in Fig. 7(b). The strong spurs in Fig. 6 are the beat signals and harmonic signals, which are mainly caused by the nonlinearity of the phase modulation and the electrical devices such as amplifiers. To suppress these spurs, other modulators such as polarization modulator (PolM) or polarization division multiplexing dual parallel MZM (PDM-MZM) can be used, to which the two oscillating frequencies can be separately applied. To achieve multi-frequency oscillation for the electrical branch, more electrical BPFs with different frequencies can be paralleled.
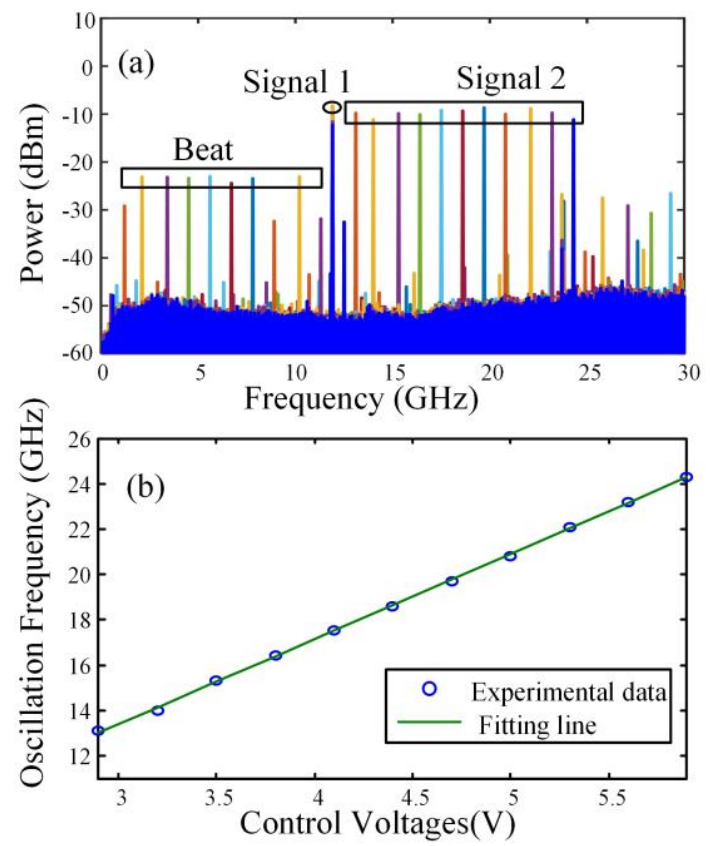

Fig. 7 (a) The superimposed spectra of the generated signals under different YIG driving voltages; (b) The relationship between the frequency of signal 2 and the driving voltage of the YIG filter.

The stability of oscillation frequency is also investigated experimentally by measuring the single sideband (SSB) phase noise. The two frequencies of the $\mathrm{OEO}$ are set at $6.60 \mathrm{GHz}$ and 22.38 GHz. Fig. 8(a) and Fig. 8(b) shows that the phase noise of signal 1 and signal 2 at $10 \mathrm{kHz}$ are $-97.38 \mathrm{dBc} / \mathrm{Hz}$ and $-112.13 \mathrm{dBc} / \mathrm{Hz}$, respectively. Some spurs that correspond to the mode 
spacing and intermodulation could be observed. Since short fiber length is adopted in the optical branch, the SSB phase noise performance is not well enough. This could be improved by adding multi-loop structure with long fiber length. Meanwhile, the MPF and BPF with narrower passband can be used to improve the stability of the generated signal.
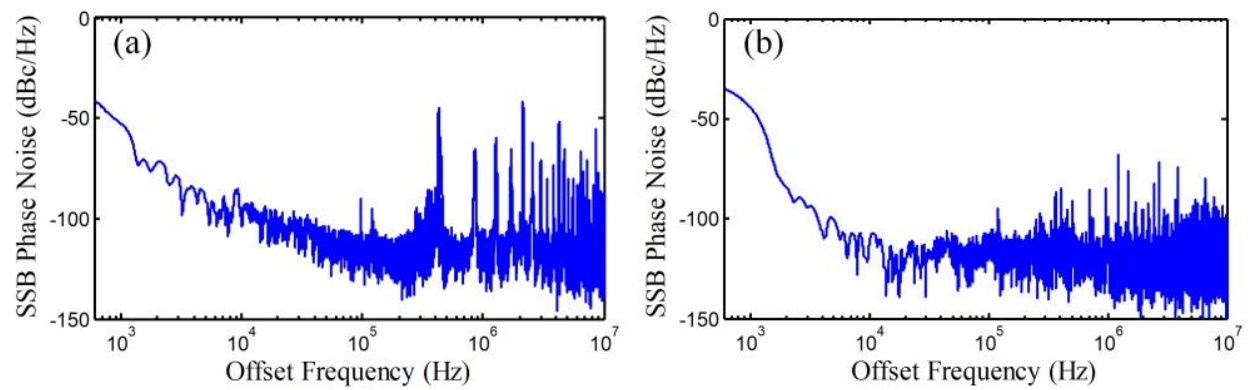

Fig. 8 (a) Measured SSB phase noise of the signal generated by the optical filtering branch at the oscillating frequency of $6.60 \mathrm{GHz}$. (b) Measured SSB phase noise of the signal generated by the electrical filtering branch at the oscillating frequency of $22.38 \mathrm{GHz}$.

\section{Conclusion}

We proposed and experimentally demonstrated a tunable multi-frequency OEO Based on a MPF and an electrical filter using PM-IM conversion. The oscillation frequencies were determined by the MPF and the electrical YIG filter. Two oscillating frequencies were generated, and independent tunability was achieved simply by changing the wavelength of the TLS or the passband of the YIG filter. By multiplexing more optical and electrical filters, the proposed OEO was capable of supporting more oscillating frequencies. The experimental results demonstrated the feasibility and tunability of proposed OEO, showing the potential application of the OEO in the field of multimode and multi-band systems, particularly for automotive radar applications.

\section{Funding}


This work was supported by the National Natural Science Foundation of China (61701532), and Natural Science Foundation of Hubei Province (2018CFB539).

\section{Declarations}

The authors declare that there are no conflicts of interest related to this article.

\section{References}

1. A. Neyer, and E. Voges, "Hybrid electro-optical multivibrator operating by finite feedback delay," Electron Letters 18, 59-60, (1982).

2. T. Berceli, and P. Herczfeld, "Microwave photonics-A historical perspective," IEEE Trans. Microw. Theory Tech 58, 2992-3000 (2010).

3. Tsuchida, and M. Suzuki, "40-Gb/s optical clock recovery using an injection-locked optoelectronic oscillator," IEEE Photonics Technology Letters 17, 211-213 (2005).

4. S. Pan, and J. P. Yao, "Multichannel optical signal processing in NRZ systems based on a frequency-doubling optoelectronic oscillator," IEEE Journal of Selected Topics in Quantum Electronics 16, 460-1468 (2010).

5. S. Pan, and J. P. Yao, "Optical clock recovery using a polarization modulator-based frequency-doubling optoelectronic oscillator," Journal of Lightwave Technology 27, 3531-3539 (2009).

6. J. Yao, "Microwave Photonics," Journal of Lightwave Technol 27, 314-335 (2009).

7. C. Y.Li, H. H.Lu, C. Y. Lin, C. A. Chu, B. R. Chen, H. H. Lin, and C. J.Wu, "Fiber-Wireless and Fiber-IVLLC Convergences Based on MZM-OEO-Based BLS," IEEE Photonics Journal 8, $1-10$ (2016).

8. P. Ghelfi, F. Laghezza, F. Scotti, et al. A fully photonics-based coherent radar system. Nature 507, 341-345 (2014). 
9. N. Majurec, "Advanced multi-frequency radar: Design, preliminary measurements and particle size distribution retrieval," Ph.D. dissertation, Dept. Elect. Comput. Eng., Massachusetts Amherst Univ., MA, USA, (2005).

10. A. Wang, J. Wo, X. Luo, Y. Wang, W. Cong, P. Du, J. Zhang, B. Zhao, J. Zhang, Yong Zhu, J. Lan, and L. Yu, "Ka-band microwave photonic ultra-wideband imaging radar for capturing quantitative target information," Opt. Express 26, 20708-20717 (2018).

11. X. Yao, and L. Maleki, "Optoelectronic microwave oscillator," Journal of The Optical Society of America B: Optical Physics 13, 1725-1735 (1996).

12. V. Jain, F. Tzeng, L. Zhou, and P. Heydari, "A Single-Chip Dual-Band 22-29-GHz/77-81GHz BiCMOS Transceiver for Automotive Radars," IEEE Journal of Solid-State Circuits 44, 3469-3485 (2009).

13. Y. Jiang, J. Liang, G. Bai, L. Hu, S. Cai, H. Li, Y. Shan, and M. Chuang, "Multifrequency optoelectronic oscillator," Optical Engineering 53, 116106 (2014).

14. F. Kong, W. Li, and J. Yao, "Transverse load sensing based on a dual-frequency optoelectronic oscillator," Optics Letters 38, 2611-2613 (2013).

15. P. Zhou, F. Zhang and S. Pan, "A tunable multi-frequency optoelectronic oscillator based on stimulated Brillouin scattering," 2015 14th International Conference on Optical Communications and Networks (ICOCN). IEEE 1-3 (2015).

16. P. Zhou, F. Zhang and S. Pan, "A multi-frequency optoelectronic oscillator based on a single phase-modulator," 2015 Conference on asers and Electro-Optics (CLEO), San Jose, CA, $1-2(2015)$. 
17. Z. Xie, S. Li, H. Yan, X. Xiao, X. Zheng, and B. Zhou, "Tunable dual frequency optoelectronic oscillator with low intermodulation based on dual-parallel Mach-Zehnder modulator," Optics Express 24, 30282-30288 (2016).

18. W. Li, and J. Yao, "A Wideband Frequency Tunable Optoelectronic Oscillator Incorporating a Tunable Microwave Photonic Filter Based on Phase-Modulation to IntensityModulation Conversion Using a Phase-Shifted Fiber Bragg Grating," IEEE Trans. Microwave Theory and Tech 60, 1735-1742 (2012).

19. W. Li, M. Li, and J. Yao, "A Narrow-Passband and Frequency-Tunable Microwave Photonic Filter Based on Phase-Modulation to Intensity-Modulation Conversion Using a PhaseShifted Fiber Bragg Grating," IEEE Trans. Microwave Theory Tech 60, 1287-1296 (2012).

20. F. Zeng, and J. Yao, "Investigation of phase-modulator-based all-optical bandpass microwave filter," Journal of Lightwave Technol 23, 1721-1728 (2005). 
Figures

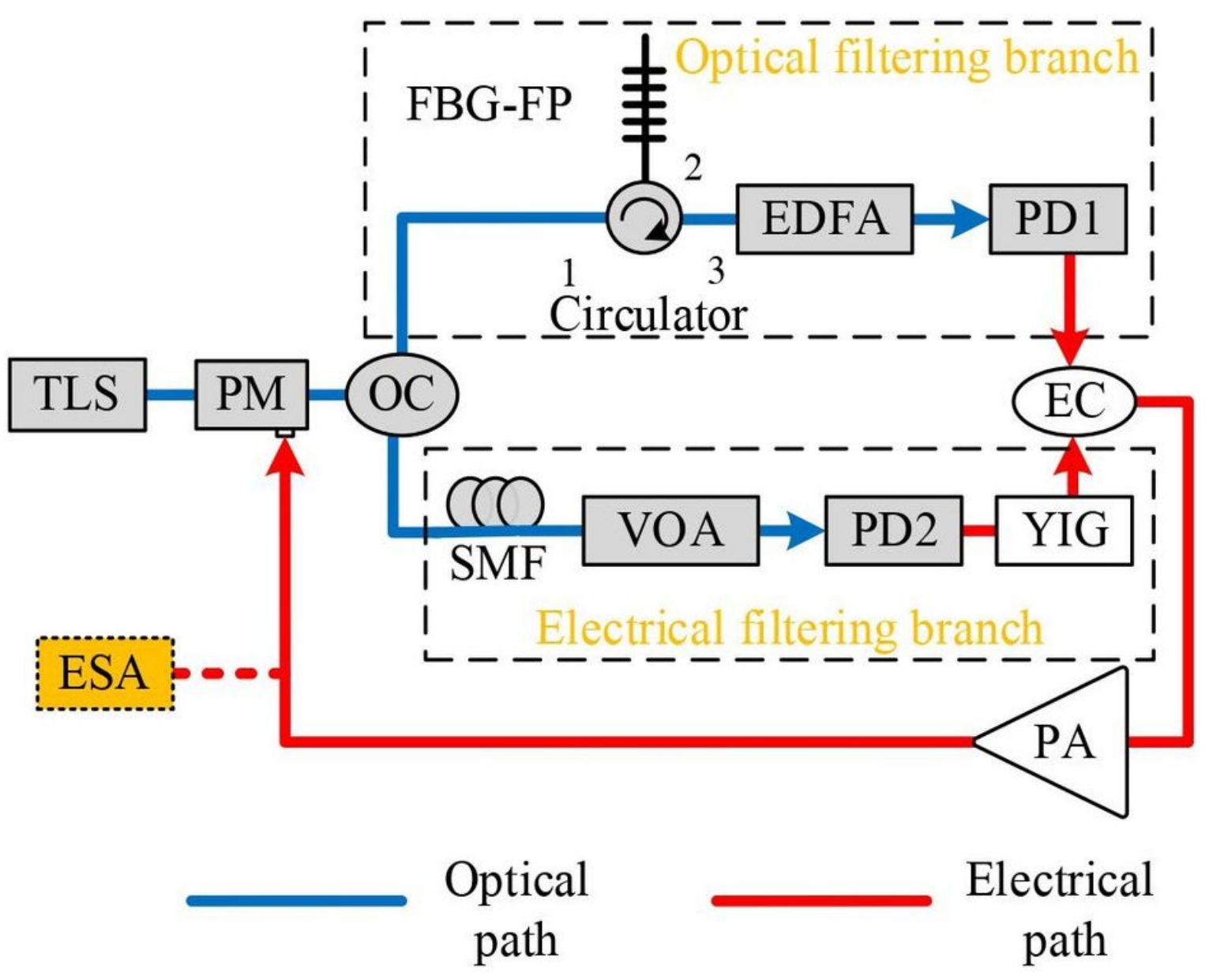

Figure 1

Schematic diagram of the proposed tunable multi-frequency OEO. 


\section{PM signal}

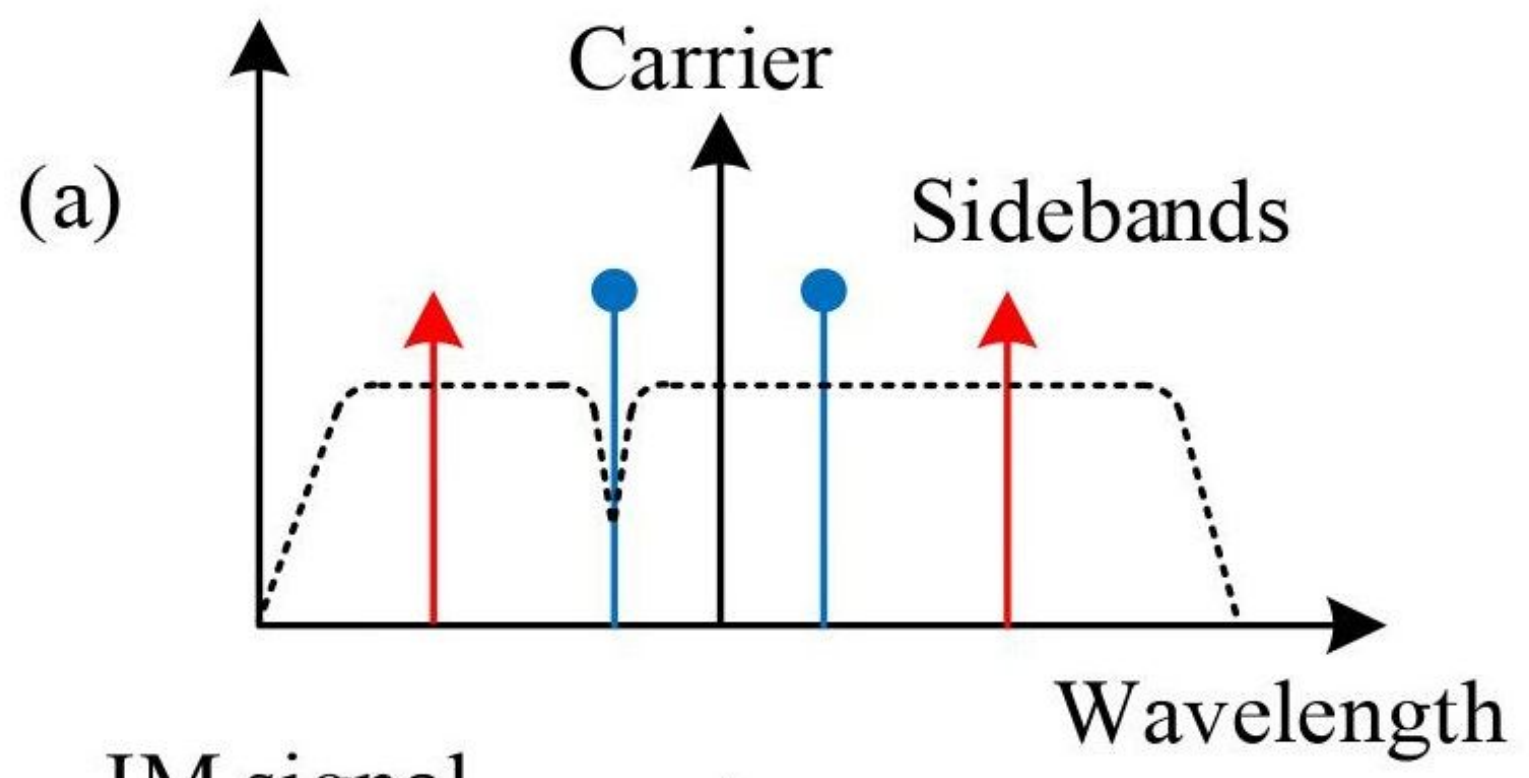

IM signal

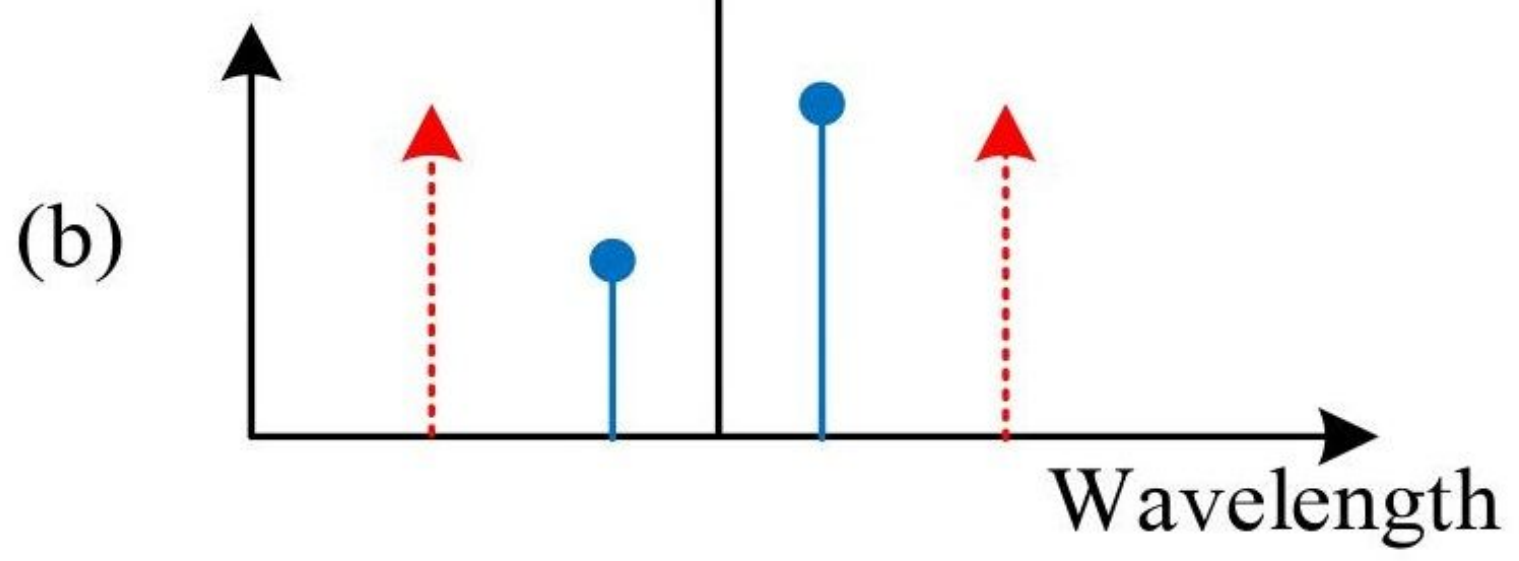

Figure 2

Operation principle of the optical filtering branch. (a) The phase-modulated signal. (b) The phasemodulated signal after filtered by FBG-FP. 


\section{Magnitude}

(a)

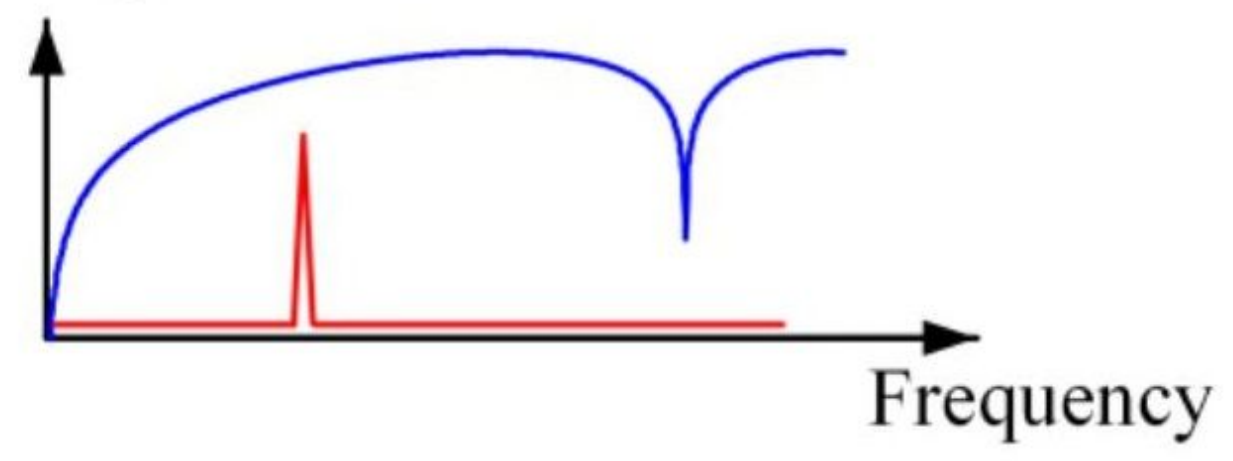

Magnitude

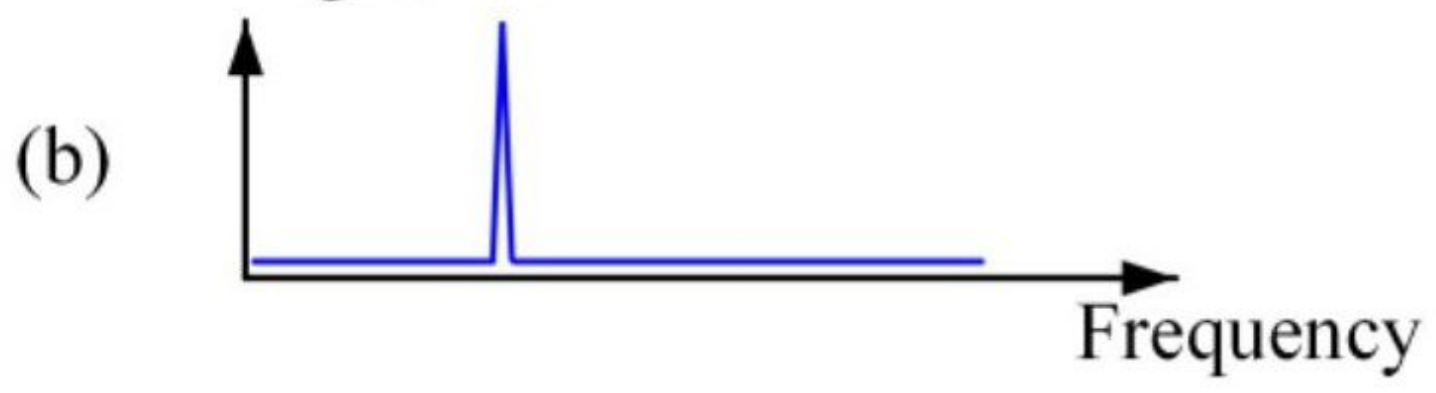

Figure 3

Operation principle of the electrical filtering branch using a YIG filter. (a) The frequency response of the band-pass MPF (the blue line) and the frequency response of the YIG filter (the red line); (b) The frequency response of the electrical filtering branch. 

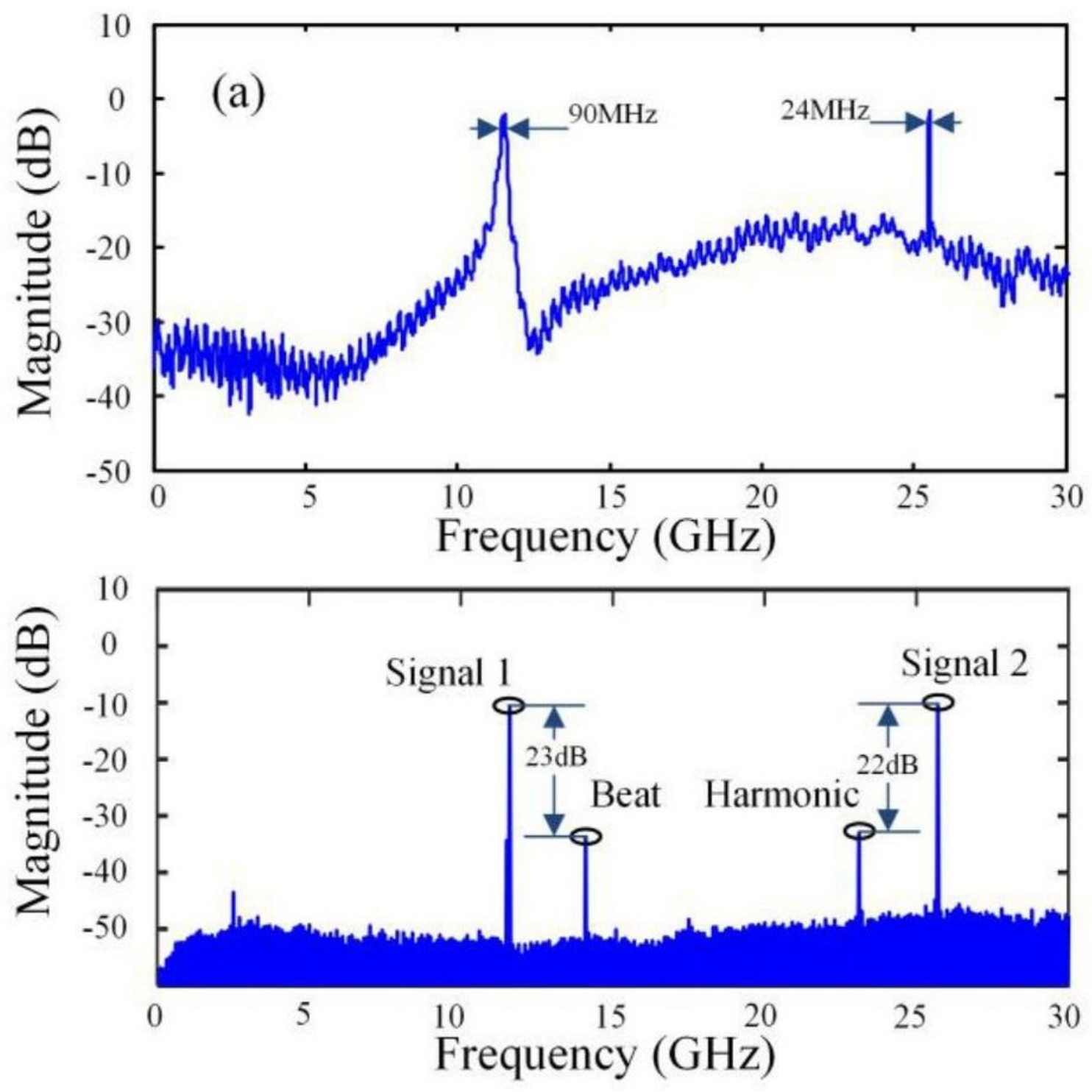

Figure 4

(a) The frequency response of the dual-frequency MPF; (b) The electrical spectrum of oscillation signals. 

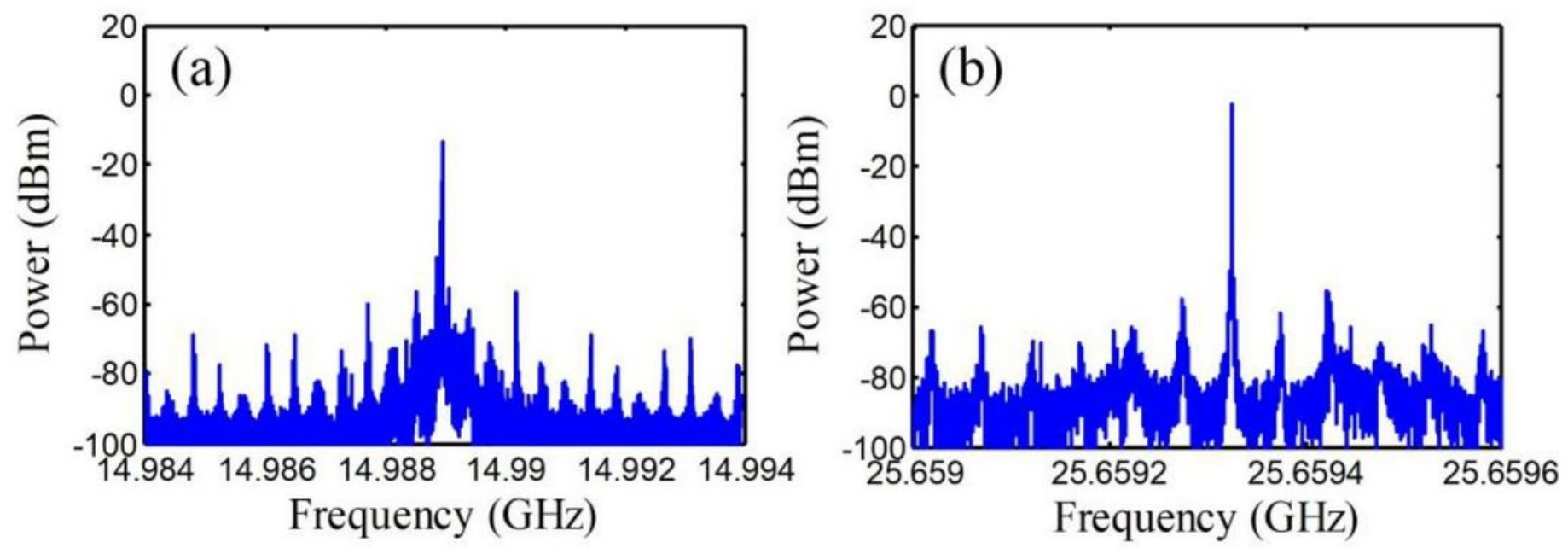

Figure 5

(a) Zoomed-in view of the oscillating frequency of the optical branch with a span of $10 \mathrm{MHz}$; (b) Zoomedin view of the oscillating frequency of the electrical branch with a span of $600 \mathrm{kHz}$; 

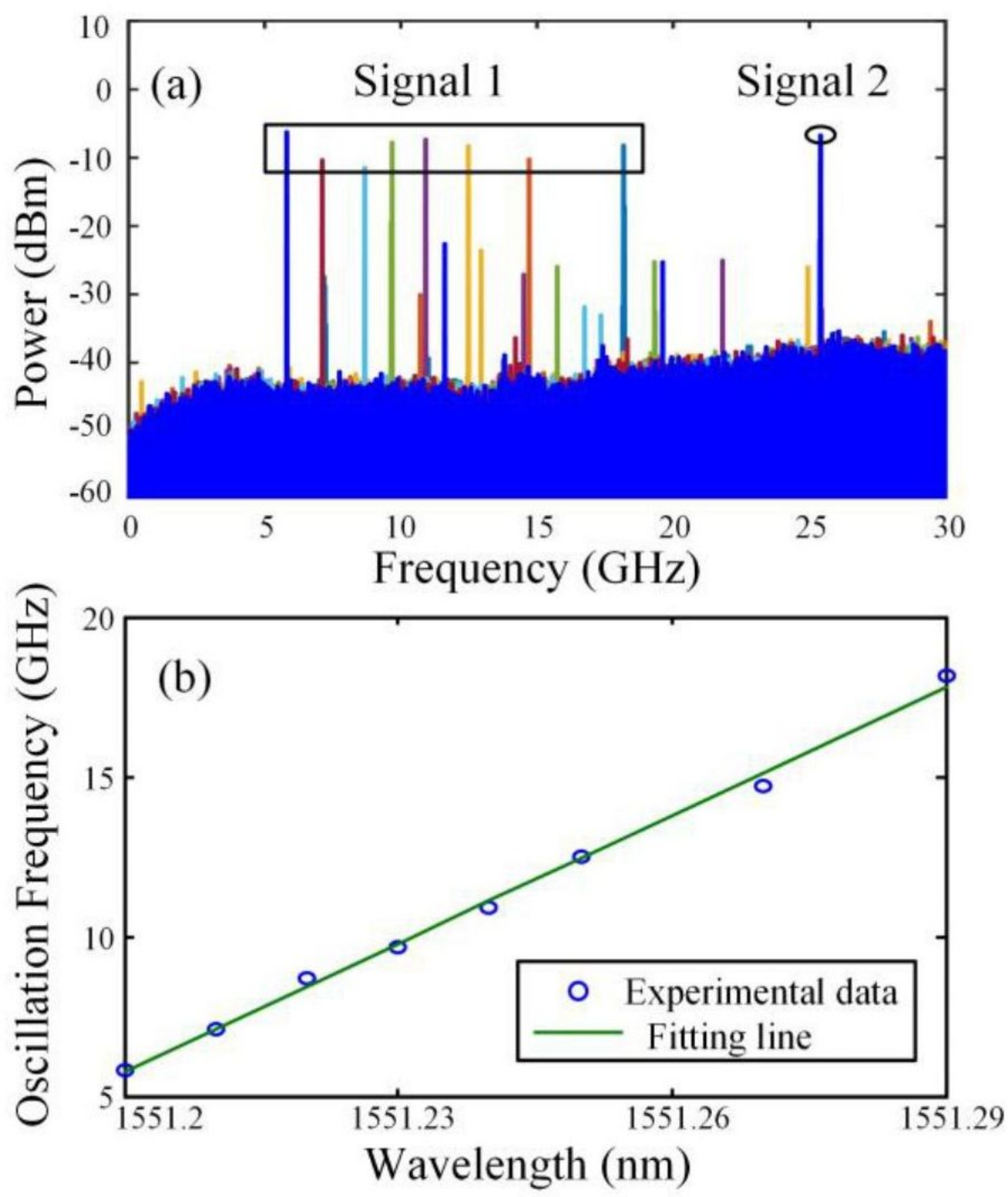

Figure 6

(a) The superimposed spectra of the generated signals under different TLS wavelengths; (b) The relationship between the frequency of signal 1 and the TLS wavelength. 

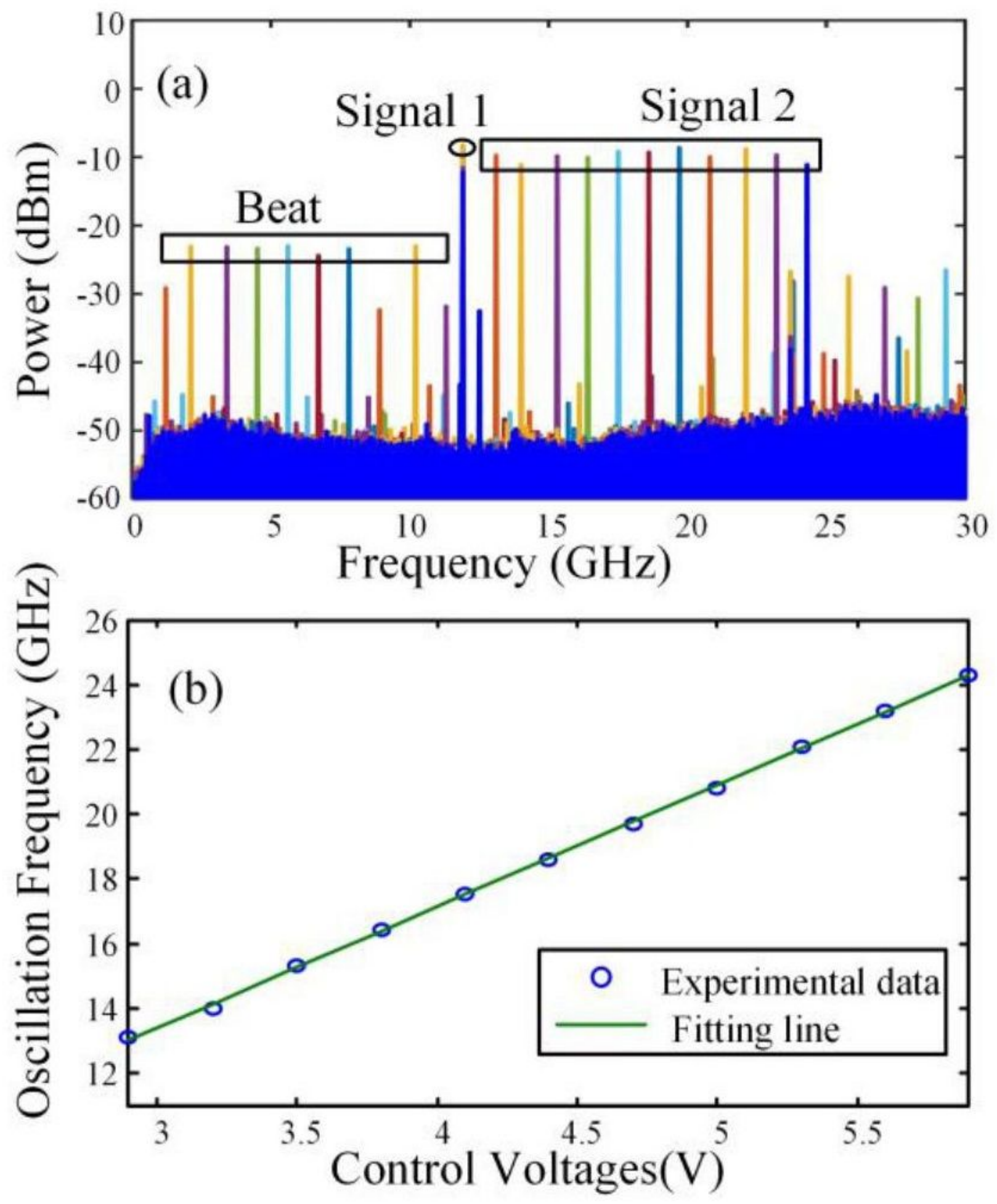

Figure 7

(a) The superimposed spectra of the generated signals under different YIG driving voltages; (b) The relationship between the frequency of signal 2 and the driving voltage of the YIG filter. 

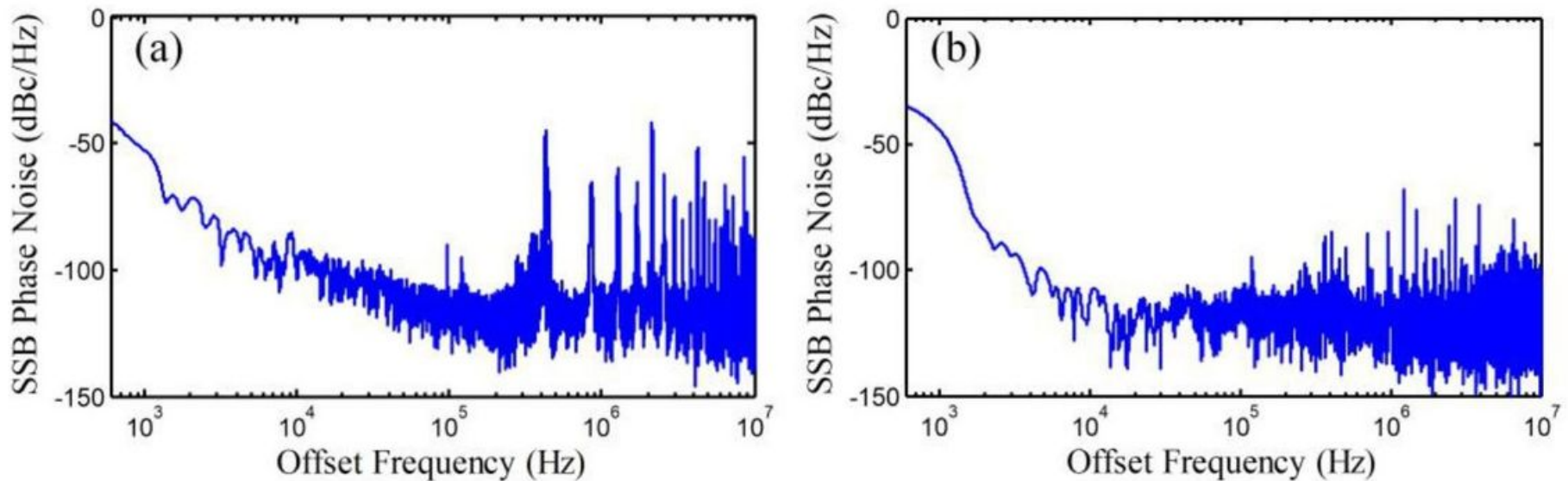

\section{Figure 8}

(a) Measured SSB phase noise of the signal generated by the optical filtering branch at the oscillating frequency of $6.60 \mathrm{GHz}$. (b) Measured SSB phase noise of the signal generated by the electrical filtering branch at the oscillating frequency of $22.38 \mathrm{GHz}$. 